\title{
ANÁLISE DA DENSIDADE ÓTIMA DE ESTRADAS FLORESTAIS EM PROPRIEDADES RURAIS
}

\author{
Flávio Cipriano de Assis do Carmo ${ }^{1}$, Nilton César Fiedler ${ }^{1 *}$, Eduardo da Silva Lopes², \\ Daniel Pena Pereira ${ }^{1}$, Heitor Broetto Marin ${ }^{1}$, Elizabeth Neire da Silva ${ }^{1}$
}

*Autor para correspondência: fiedler@pq.cnpq.br

RESUMO: Conduziu-se este estudo, com o objetivo de analisar a densidade de estradas em propriedades rurais e comparar com o calculo da densidade ótima em áreas acidentadas em empresas florestais no sul do Espírito Santo. O trabalho foi executado em seis pequenas propriedades rurais, baseando-se nos custos de estradas de uso florestal, extração de madeira e nos custos de perda de área produtiva. A análise técnica englobou estudo de tempos e movimentos e de produtividade. A análise econômica englobou custos operacionais, custos de produção e rendimentos para diferentes cenários de produtividade $\left(180 \mathrm{~m}^{3} \cdot \mathrm{ha}^{-1}, 220 \mathrm{~m}^{3} \cdot h \mathrm{~h}^{-1}\right.$ e $\left.250 \mathrm{~m}^{3} \cdot \mathrm{ha}^{-1}\right)$. De acordo com os resultados, as propriedades possuem densidades de estradas muito acima do ótimo, o que reflete a falta de critérios no planejamento dos talhões, resultando num pior aproveitamento da área de plantio e elevados custos de produção. A propriedade 1 apresentou a maior densidade de estradas $\left(373,92 \mathrm{~m} \cdot \mathrm{ha}^{-1}\right)$ e a propriedade 5 apresentou a menor densidade $\left(111,56 \mathrm{~m} \cdot \mathrm{ha}^{-1}\right)$.

Palavras-chave: Colheita florestal, operações florestais, análise econômica.

\section{ANALYSIS OF OPTIMUM DENSITY OF FOREST ROADS IN RURAL PROPERTIES}

\begin{abstract}
This study analyzed the density of roads in rural properties in the south of the Espirito Santo and compared it with the calculation of the optimal density in forestry companies in steep areas. The work was carried out in six small rural properties based on the costs of roads of forest use, wood extraction and the costs of loss of productive area. The technical analysis included time and movement study and productivity. The economic analysis included operational costs, production costs and returns for different scenarios of productivity $\left(180 \mathrm{~m} \cdot \mathrm{ha}^{-1}, 220 \mathrm{~m} \cdot \mathrm{ha}^{-1}\right.$ and $\left.250 \mathrm{~m} \cdot \mathrm{ha^{-1 }}\right)$. According to the results, all the properties have densities of road well above the optimum, which reflects the lack of criteria in the planning of the forest stands, resulting in a inadequate use of plantation area. Property 1 had the highest density of roads $\left(373.92 \mathrm{~m}^{\left.-h \mathrm{a}^{-1}\right)}\right.$ and the property 5 presented the lowest density $\left(111.56 \mathrm{~m} \cdot \mathrm{ha} \mathrm{s}^{-1}\right)$.
\end{abstract}

Key words: Forest harvesting, forest operations, economic analysis.

\section{INTRODUÇÃO}

Nos empreendimentos florestais que empregam a madeira como fonte de matéria-prima é de fundamental importância o planejamento minucioso de todas as atividades envolvidas no processo produtivo, resultando em racionalização da produção, melhoria da produtividade e redução dos custos operacionais (HOSOKAWA; MENDES, 1984). Além disso, é necessário escolher com critérios o local onde serão localizados os plantios florestais, principalmente em termos de distância até a indústria, pois o transporte representa a maior parcela significativa dos custos da madeira colocada na indústria (ARCE, 1997; MACHADO; LOPES, 2002).

As estradas florestais são as mais importantes vias de acesso às florestas, servindo para viabilizar o tráfego de mão-de-obra e os meios de produção necessários para o investimento florestal. Segundo Zagonel et al. (2008), a atividade de extração de madeira irá determinar

${ }^{1}$ Universidade Federal do Espírito Santo - Alegre, Espírito Santo, Brasil

${ }^{2}$ Universidade Estadual do Centro-Oeste - Irati, Paraná, Brasil o dimensionamento ótimo das estradas florestais, em função, principalmente, das características dos métodos (manual ou mecanizado) a serem utilizados e do relevo dos talhões. À medida que se diminui a distância de extração da madeira, eleva-se a densidade de estradas, resultando em baixos custos de extração. No entanto, os custos com construção e manutenção de estradas serão maiores, em decorrência dessa maior densidade, além de contribuir para a redução da área produtiva e maior possibilidade de degradação ambiental.

Dessa forma, é muito importante determinar o ponto ótimo entre a densidade de estradas a ser construída e as distâncias de arraste, de acordo com as condições do terreno e estrutura da floresta para melhorar a eficiência das operações e otimização das áreas de plantio. Becker (1994) considera a construção das estradas como o maior investimento na colheita florestal, sendo que o planejamento da malha viária deve ser feito por pessoal qualificado, baseado nas necessidades da empresa.

Cerne, Lavras, v. 19, n. 3, p. 451-459, jul./set. 2013 
A densidade de estradas, expressa em m.ha ${ }^{-1}$ (metros lineares por hectare) pode variar de acordo com a intensidade da atividade florestal, com as condições do terreno e com o sistema de colheita de madeira adotado. A densidade ótima de estradas tende a ser maior, na medida em que a declividade do terreno aumenta, tendo grande importância para caracterizar a intensidade da construção de estradas e indicando a viabilidade da malha viária planejada (EMPRESA BRASILEIRA DE PESQUISA AGROPECUÁRIA-EMBRAPA, 1997; LOPES et al., 2002).

Objetivou-se, com esta pesquisa, analisar a densidade ótima de estradas florestais em propriedades rurais na região sul do Espírito Santo, visando à racionalização do binômio estradas e extração florestal.

\section{MATERIAL E MÉTODOS}

\section{1. Área de Estudo}

A pesquisa foi realizada em áreas de colheita florestal de seis propriedades rurais, localizadas nos municípios de Vargem Alta (propriedades 1 e 2), Alegre (propriedade 3), Cachoeiro de Itapemirim (propriedade 4), São José do Calçado (propriedade 5) e Jerônimo Monteiro (propriedade 6), todas localizadas no Sul do estado do Espírito Santo. A espécie plantada era o híbrido das espécies Eucalyptus grandis e Eucalyptus urophilla, em espaçamento $3 \times 3$ metros, produzidos por propagação vegetativa. Todos os plantios estavam localizados em áreas declivosas, com inclinação aproximada de $35 \%$, onde não era viável a mecanização das operações. A madeira produzida era destinada a celulose e todas as propriedades participavam de contrato de fomento florestal com uma empresa florestal.

\subsection{Coleta de dados}

A coleta de dados foi realizada de agosto de 2010 a maio de 2011. Inicialmente, foi realizada uma visita às propriedades rurais, onde foi utilizado um questionário para a identificação dos seguintes atributos: tamanho territorial da propriedade, características das espécies plantadas, relevo da área e ano de construção e manutenção das estradas.

Com o auxilio de um GPS de navegação de marca GARMIN, modelo GPSMAP $60 \mathrm{CSx}$, foram coletados os pontos no entorno das estradas florestais, sendo, posteriormente, os dados transferidos para um Sistema de Informação Geográfica para as medições da largura e distâncias entre as estradas, medições das áreas dos polígonos de plantio, realização do mapeamento e cálculo das densidades de estradas florestais.

Cerne, Lavras, v. 19, n. 3, p. 451-459, jul./set. 2013
Em seguida, de posse dessas informações, calculouse a densidade das estradas secundárias existentes nas propriedades, comparando-a com uma situação desejável para as condições brasileiras que, segundo Machado (1989), deve ser 90,1 metros lineares de estradas por hectare de área plantada. A densidade ótima de estradas foi determinada por equações específicas em que a extração era realizada de forma manual, seguindo metodologia de Zagonel (2005).

\subsection{Sistema de colheita de madeira}

O sistema de colheita de madeira adotado nas propriedades rurais foi o de toras curtas ("cut-to-lenght"), onde a derrubada, desgalhamento, destopamento e traçamento da madeira em toras de 3 metros era feito com o uso de motosserras, enquanto a extração era realizada por tombamento manual até a margem das estradas florestais.

\subsection{Determinação do ciclo operacional de extração das atividades efetivas - equação específica}

O tempo efetivo consumido por ciclo operacional da atividade de extração foi expresso pela seguinte equação:

$\mathrm{ae}=\mathrm{VV}+\mathrm{FC}+\mathrm{VC}+\mathrm{DC}$ (Equação 1)

em que:

ae = tempo efetivo de extração;

$\mathrm{VV}=$ tempo de viagem descarregado;

$\mathrm{FC}=$ tempo de carregamento;

$\mathrm{VC}=$ tempo de viagem carregado; $\mathrm{e}$

$\mathrm{DC}=$ tempo de descarregamento $(\mathrm{min})$.

Por definição tem-se a velocidade expressa pela equação abaixo:

velocidade $=\frac{\text { distância }}{\text { tempo }}$

(Equação 2)

em que:

VSC $=$ velocidade média de extração sem carga $\left(\mathrm{m} \mathrm{min}^{-1}\right)$;

$\mathrm{VCC}=$ velocidade média de extração com carga $\left(\mathrm{m} \cdot \mathrm{min}^{-1}\right)$;

DME = distância média de extração (m).

Desta forma, podemos dizer:

$\mathrm{VV}=\frac{\mathrm{DME}}{\mathrm{VSC}}$

(Equação 3)

$\mathrm{VC}=\frac{\mathrm{DME}}{\mathrm{VCC}}$

(Equação 4) 
Para a determinação da velocidade média de extração procedeu-se o estudo de tempos e movimentos pelo método contínuo, com auxilio de um cronômetro digital centesimal e uma trena de 25 metros para medição da distância de extração.

\subsection{Distância média de extração (DME)}

Malinovski e Perdoncini (1990) expressaram a relação entre a densidade de estradas e a distância média de extração como:

$\mathrm{DME}=\frac{2500 * \mathrm{~T} * \mathrm{~V}}{\mathrm{DOE}}$

em que:

$\mathrm{DOE}=$ densidade ótima de estradas

$\mathrm{T}=$ fator de correção para a extração, para casos em que a extração não é feita em linha reta e perpendicular à estrada e não termina no ponto mais próximo da origem;

$\mathrm{V}=$ fator de correção para rede de estradas, utilizado quando as estradas são tortuosas e não paralelas, com espaçamento diferente entre as mesmas.

Neste estudo, foi utilizado o fator de correção estimado em 1,55 ( $\left.\mathrm{V}^{*} \mathrm{~T}\right)$ valor adotado por Souza (2001), que utilizou fatores como 1,45 para terrenos planos e 1,55 para terrenos ondulados. Substituindo os valores na fórmula da DME tem-se:

$\mathrm{DME}=\frac{2500 * 1,55}{\mathrm{DOE}} \rightarrow \mathrm{DME}=\frac{3875}{\mathrm{DOE}}$

(Equação 6)

\subsection{Custo de estradas de uso florestal}

O custo de estradas foi obtido em função da DOE, utilizando incremento médio anual da floresta e o custo anual de estradas, aplicando-se a seguinte expressão (PEREIRA NETO, 1995):

$\mathrm{C}_{\mathrm{est}}=\frac{\mathrm{CAe}}{\mathrm{IMA}} * \mathrm{DOE}$

em que:

Cest $=$ custo de estradas $\left(\mathrm{R} \$ . \mathrm{m}^{-3}\right)$;

$\mathrm{CAe}=$ custo anual de estradas florestais $\left(\mathrm{R} \$ . \mathrm{m}^{-1}\right)$;

IMA $=$ incremento médio anual $\left(\mathrm{m}^{3} \cdot \mathrm{ha}^{-1}\right)$.

O valor do IMA adotado para os diferentes cenários de produtividades foram 30,$0 ; 36,7$ e $41,7 \mathrm{~m}^{3} / \mathrm{ha} / \mathrm{ano}$, sendo os valores utilizados pela empresa fomentadora das propriedades rurais fomentadas e aplicados para as demais propriedades avaliadas. Para a determinação do custo anual de estradas, consideraram-se os custos de construção e de manutenção, sendo o custo de construção obtido por meio das produtividades médias das máquinas e dos custos operacionais dos recursos utilizados nas operações de construção, sendo utilizada a seguinte expressão:

$\mathrm{Cc}=\frac{\sum(\operatorname{Pr} * \mathrm{COr})}{1000}$

(Equação 8)

em que:

$\mathrm{Cc}=$ custo de construção por metro linear de estrada $\left(\mathrm{R} \$ . \mathrm{m}^{-1}\right)$;

$\operatorname{Pr}=$ produtividade média do recurso (máquinas e veículos), utilizados na construção (h. $\left.\mathrm{Km}^{-1}\right)$;

$\mathrm{COr}=$ custo operacional do recurso $\left(\mathrm{R} \$ \cdot \mathrm{h}^{-1}\right)$. adotou-se um custo de RS3,23.h $\mathrm{h}^{-1}$ segundo Pereira (2010).

Baseado em Moosmayer (1967) e Speidel (1966), representou-se o custo de construção numa base anual, por meio da depreciação linear das estradas e dos juros do capital investido na construção. Na determinação da depreciação linear, utilizou-se a seguinte fórmula:

$\mathrm{D}=\frac{\mathrm{Cc}}{\mathrm{V}}$

(Equação 9)

em que:

$\mathrm{D}=$ depreciação anual por metro linear de estrada $\left.\mathrm{R} \$ . \mathrm{m}^{-1}\right)$; $\mathrm{V}=$ vida útil da estrada (anos).

Segundo Becker (1994) e Pereira Neto (1995), o período para determinar a depreciação das estradas florestais varia entre 20 e 30 anos. Dessa forma, utilizouse no calculo da depreciação, uma vida útil de 21 anos que representa uma duração média das estradas para três rotações de povoamento. Na obtenção de juros do capital investido, aplicou-se a seguinte fórmula:

$\mathrm{J}=\frac{\mathrm{Cc}}{2} * \frac{\mathrm{i}}{100}$

(Equação 10)

em que:

$\mathrm{J}=$ juros anuais por metro linear de estrada $\left(\mathrm{R} \$ . \mathrm{m}^{-1}\right)$;

$\mathrm{i}=$ taxa anual de juros (\%).

A taxa de juros que será adotada será de $4 \%$ ao ano, considerando que esse valor é frequentemente utilizado nas empresas florestais. Para a determinação do custo anual de manutenção de estradas $(\mathrm{Cm})$, utilizaram-se os custos operacionais e as produtividades médias dos recursos utilizados nas operações de manutenção anual das estradas, de acordo com a fórmula:

$\mathrm{Cm}=\frac{\sum(\operatorname{Pr} * \mathrm{COr})}{1000}$

(Equação 11)

Cerne, Lavras, v. 19, n. 3, p. 451-459, jul./set. 2013 


\subsection{Custo por perda de área produtiva (PAP)}

$\mathrm{Na}$ obtenção do custo da perda de área produtiva, em função da densidade de estradas, utilizou-se o incremento médio anual dos plantios e o custo anual da perda de área por metro linear de estrada, segundo a fórmula apresentada por Pereira Neto (1995):

$\mathrm{Cpap}=\frac{\mathrm{CAp}}{\mathrm{IMA}} * \mathrm{DOE}$

(Equação 12)

em que:

Cpap = custo de perda de área produtiva $\left(\mathrm{R} \$ . \mathrm{m}^{-3}\right)$;

Cap = custo anual de perda de área de produção por metro linear de estrada $\left(\mathrm{R} \$ . \mathrm{m}^{-1}\right)$.

Na determinação do custo anual de perda de área produtiva, considerou-se que este representa um custo de oportunidade (PEREIRA NETO, 1995). O custo de oportunidade de um determinado produto, de acordo com Leftwich (1983), representa o valor dos produtos alternativos renunciados que seria possível obter com os recursos considerados. Na obtenção desse custo, utilizouse a largura da faixa de estrada (distância entre sarjetas), o incremento médio anual da floresta e o valor médio da madeira em pé, de acordo com a fórmula:

Cap $=\frac{\mathrm{A}}{10.000} * \mathrm{Vm}$

(Equação 13)

em que:

$\mathrm{A}=$ área de estrada por metro linear $\left(\mathrm{m}^{2} \cdot \mathrm{m}^{-1}\right)$;

$\mathrm{Vm}=$ valor médio da madeira em pé $\left(\mathrm{R} \$ \mathrm{~m}^{-3}\right)$.

$\mathrm{O}$ valor médio de madeira em pé foi considerado a $\mathrm{R} \$ 40,00 \mathrm{~m}^{-3}$ (valor médio na região). Foram considerados cenários de produtividades de 180, 220 e $250 \mathrm{~m}^{3}$.ha ${ }^{-1}$, para um valor da madeira do híbrido Eucalyptus grandis $x$ Eucalyptus urophylla em pé igual a $\mathrm{R} \$ 40,00 . \mathrm{m}^{-3}$.

\subsection{Custo de extração da madeira}

O custo de extração da madeira foi calculado com base no custo operacional de cada equipamento e na produtividade da operação, em função da distância média de extração, aplicando a expressão abaixo,conforme metodologia de Pereira Neto (1995):

$\mathrm{Cext}=\frac{\mathrm{CO}}{\mathrm{Pr}}$

(Equação 14)

em que:

Cext $=$ custo de extração florestal $\left(\mathrm{R} \$ . \mathrm{m}^{-3}\right)$;

$\mathrm{CO}=$ custo operacional do funcionário $\left(\mathrm{R} \$ \cdot \mathrm{h}^{-1}\right)$,

$\operatorname{Pr}=$ produtividade média efetiva da operação $\left(\mathrm{m}^{3} \cdot \mathrm{h}^{-1}\right)$.
A produtividade média efetiva da operação de extração foi determinada pela razão entre o volume médio extraído por ciclo e a equação das atividades efetivas em função da distância média de extração, segundo a fórmula expressa a seguir:

$\operatorname{Pr}=\frac{\text { Vméd }}{\text { ae }} * 60$

(Equação 15)

em que:

$\operatorname{Pr}=$ produtividade média efetiva da operação $\left(\mathrm{m}^{3} \cdot \mathrm{ha}^{-1}\right)$;

Vméd= volume médio extraído por ciclo $\left(\mathrm{m}^{3}\right)$;

$\mathrm{ae}=$ equação das atividades efetivas (min).

Baseado nas equações das atividades efetivas, foi calculado o custo de extração, ao substituir a produtividade média efetiva da operação no modelo do custo de extração florestal obteve-se:

Cest $=\frac{\mathrm{CO}}{60 * \text { Vméd }} * \mathrm{ae}$

(Equação 16)

\subsection{Cálculo da densidade ótima de estradas em função dos custos totais}

A DOE foi calculada conforme modelo de Dietz (1983), pela seguinte fórmula:

$\mathrm{CT}=\mathrm{Cest}+\mathrm{Cext}+\mathrm{Cpap}$

(Equação 17)

\subsection{Densidade ótima de estradas}

A densidade ótima de estradas foi obtida em função da derivada dos custos totais, obtendo a seguinte expressão:

$0=C^{\prime}$ est $+C^{\prime}$ pap $+C^{\prime}$ ext

(Equação 18)

\section{RESULTADOS E DISCUSSÃO}

Para a determinação do ciclo operacional das atividades efetivas de extração de madeira, ao substituirmos a equação 3 e 4 na equação 1 tem-se:

$\mathrm{ae}=\frac{\mathrm{DME}}{\mathrm{VSC}}+\mathrm{FC}+\frac{\mathrm{DME}}{\mathrm{VCC}}+\mathrm{DC}$

(Equação 19)

$\mathrm{Na}$ coleta dos dados, foi encontrada para todas as propriedades avaliadas uma produtividade média de extração manual por ciclo de $0,02 \mathrm{~m}^{3}$ (para toras de 3 metros), além dos tempos médios de formação de carga (FC) de 0,1 min.ciclo ${ }^{-1}$, de descarga (DC) de 0,07 min.ciclo $^{-1}$, velocidade média sem carga (VSC) de $66,67 \mathrm{~m} \cdot \mathrm{min}^{-1}$, velocidade média com carga (VCC) de $30 \mathrm{~m} \cdot \mathrm{min}^{-1}$.

Cerne, Lavras, v. 19, n. 3, p. 451-459, jul./set. 2013 
Assim, substituindo esses resultados com a equação 6 e 19, obteve a seguinte equação:

$\mathrm{ae}=0,17+\frac{187,87}{\mathrm{DOE}}$

(Equação 20)

\subsection{Custos de estradas de uso florestal}

O custo anual com estradas primárias foi de $\mathrm{R} \$ 0,73$ por metro linear, sendo esse custo determinado por meio do custo médio regional de construção de $\mathrm{R} \$ 3.990,00$ por km e manutenção de R \$ 460,00 por Km. Zagonel (2005), em condições de áreas planas, obteve um custo anual de $\mathrm{R} \$ 2,54$ por metro linear, enquanto Souza (2001) obteve $\mathrm{R} \$ 1,37$ e Pereira Neto (1995) R \$1,42 por metro linear de estradas. $\mathrm{O}$ fato do valor ter sido menor ao encontrado pelos autores está relacionado com a menor largura das estradas das propriedades estudas (4,5 metros) em relação aos outros trabalhos (8 metros), aumentando, assim, o rendimento da operação e acarretando num menor custo final de estrada. Porém, em razão de as propriedades apresentarem estradas com menor largura, o transporte de madeira se restringe a veículos de pequeno porte, que podem ocasionar custos maiores para os produtores nessa etapa. Na Figura 1, apresentam-se os custos anuais de estradas primárias obtidos neste estudo e os percentuais em relação aos custos totais.

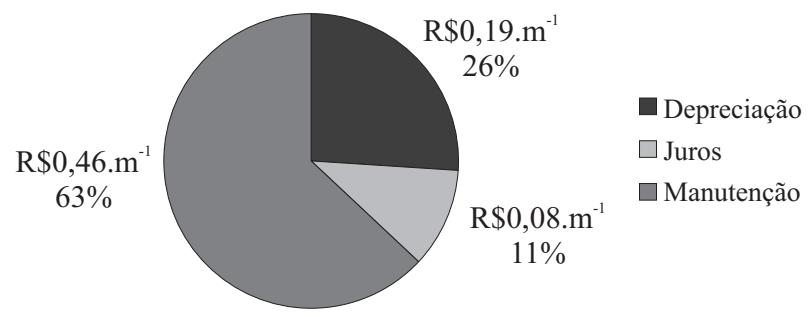

Figura 1 - Custos anuais de estradas de uso florestal e percentual em relação aos totais.

Figure 1 - Annual costs of roads of forest use and percent in relation of a cost total.

Dessa forma, foi obtido o custo anual de estradas florestais por meio do somatório da depreciação, juros e custo anual de manutenção, que resultou num custo de $\mathrm{R} \$ 0,73$. metro linear ${ }^{-1}$ de estrada florestal.A partir desse custo anual, obteve-se a equação do custo de estradas primárias e secundárias ( $\mathrm{R} \$$.metro linear $\left.{ }^{-1}\right)$, sendo que, para determinação dos custos das estradas secundárias, adotouse o custo de $33 \%$ das estradas primárias (ZAGONEL, 2005), conforme apresentado na Tabela 1. Porém como
Tabela 1 - Custo de estradas para diferentes cenários de produtividade.

Table 1 - Cost of roads for different scenes of productivity.

\begin{tabular}{lccc}
\hline & \multicolumn{3}{c}{ Cenários de Produtividade $\left(\mathrm{m}^{3} \cdot \mathrm{ha}^{-1} \cdot \mathrm{ano}^{-1}\right)$} \\
\cline { 2 - 4 } & 30,0 & 36,7 & 41,7 \\
\hline $\begin{array}{l}\text { Custos Estrada } \\
\text { Primária }\end{array}$ & $0,0243 * \mathrm{DOE}$ & $0,0199 * \mathrm{DOE}$ & $0,0175^{*} \mathrm{DOE}$ \\
$\begin{array}{l}\text { Custos Estrada } \\
\text { Secundária }\end{array}$ & $0,0081 * \mathrm{DOE}$ & $0,0066^{*} \mathrm{DOE}$ & $0,0058^{*} \mathrm{DOE}$ \\
\hline
\end{tabular}

neste estudo buscou a DOE em função da extração da madeira, todos os custos foram determinados a partir da densidade das estradas secundárias.

\subsection{Custos por perda de área produtiva (PAP)}

A largura da faixa de estrada, ou seja, da plataforma (leito) mais as sarjetas, variou nas propriedades. Dessa forma, foi elaborada a Tabela 2 que mostra o custo de perda de área produtiva em função da largura das estradas secundárias.

Tabela 2 - Custo da perda de área produtiva (Cpap) em relação às faixas das estradas.

Table 2 - Cost of the loss of productive area in relation to the tracks of roads.

\begin{tabular}{ccc}
\hline Propriedade & $\begin{array}{c}\text { Faixa média de largura } \\
\text { das estradas }(\mathrm{m})\end{array}$ & Equação \\
\hline 1 & 3,25 & Cpap $=0,0221 * \mathrm{DOE}$ \\
2 & 3,65 & Cpap $=0,0248 * \mathrm{DOE}$ \\
3 & 3,80 & Cpap $=0,0268 * \mathrm{DOE}$ \\
4 & 4,05 & Cpap $=0,0276 * \mathrm{DOE}$ \\
5 & 3,55 & Cpap $=0,0242 * \mathrm{DOE}$ \\
6 & 3,80 & Cpap $=0,0258 * \mathrm{DOE}$ \\
\hline
\end{tabular}

\subsection{Custos de extração da madeira}

Para a determinação do custo de extração da madeira,adotou-se um custo operacional do recurso de RS3,23.h ${ }^{-1}$, segundo Pereira (2010) e uma produtividade média de extração manual por ciclo de $0,02 \mathrm{~m}^{3}$ medido nas propriedades.

Assim, foi possível determinar a equação de custo de extração em função da densidade ótima de estradas, conforme os modelos adaptados por equipe de colheita.

Cext $=0,4437+\frac{490,34}{\mathrm{DOE}}$

(Equação 21)

Cerne, Lavras, v. 19, n. 3, p. 451-459, jul./set. 2013 


\subsection{Cálculo da densidade ótima de estradas em função dos custos totais}

A minimização dos custos totais (CT) é atendida matematicamente pela derivada do custo global em função da densidade de estradas. Assim, a densidade ótima de estradas foi determinada pela derivada das equações dos custos globais (equação 16). Na Tabela 3, representamse as derivadas dos custos de perda de área, estradas e extração.
Ao substituir as derivadas dos custos de estradas, por perda de área produtiva e extração foram encontradas as equações de densidades de estradas (Tabela 4).

A partir das equações da Tabela 4, obteve os resultados da densidade ótima de estradas (DOE) e da distância média de extração (DME), para as seis propriedades analisadas. Ao aplicar os respectivos valores de DOE nas equações, obtiveram-se os custos específicos de estradas, perda de área produtiva e de extração. $\mathrm{O}$

Tabela 3 - Custos de perda de área produtiva (Cpap), estradas (Cest) e de extração (Cext) utilizados na determinação da densidade ótima de estradas.

Table 3 - Costs of loss of productive area, roads and extraction used in determining the optimal density of roads.

\begin{tabular}{|c|c|c|c|c|c|c|c|c|c|}
\hline \multirow{3}{*}{ Propriedades } & \multicolumn{3}{|c|}{ C'pap } & \multicolumn{3}{|c|}{ C'est } & \multicolumn{3}{|c|}{ C'ext } \\
\hline & \multicolumn{9}{|c|}{ Produtividade $\left(\mathrm{m}^{3} \cdot \mathrm{ha}^{-1}\right)$} \\
\hline & 180 & 220 & 250 & 180 & 220 & 250 & 180 & 220 & 250 \\
\hline 1 & & 0,0221 & & & & & & & \\
\hline 2 & & 0,0248 & & & & & & & \\
\hline 3 & & 0,0268 & & 0 & 000 sca & & & 490,34 & \\
\hline 4 & & 0,0276 & & 0,0081 & 0,00003 & 0,00583 & & $\overline{\mathrm{DOE}^{2}}$ & \\
\hline 5 & & 0,0242 & & & & & & & \\
\hline 6 & & 0,0258 & & & & & & & \\
\hline
\end{tabular}

Tabela 4 - Equações da derivada dos custos globais para determinação da DOE.

Table 4 - Equations derived from the global costs to determine the optimal density road.

\begin{tabular}{cccc}
\hline \multirow{2}{*}{ Propriedade } & Produtividade $\left(\mathrm{m}^{3} \cdot \mathrm{ha}^{-1}\right)$ & 220 \\
\cline { 2 - 4 } 1 & $0=0,0221+0,0081-\frac{490,34}{\mathrm{DOE}^{2}}$ & $0=0,0221+0,0066-\frac{490,34}{\mathrm{DOE}^{2}}$ & $0=0,0221+0,0058-\frac{490,34}{\mathrm{DOE}^{2}}$ \\
\hline 3 & $0=0,0248+0,0081-\frac{490,34}{\mathrm{DOE}^{2}}$ & $0=0,0248+0,0066-\frac{490,34}{\mathrm{DOE}^{2}}$ & $0=0,0248+0,0058-\frac{490,34}{\mathrm{DOE}^{2}}$ \\
4 & $0=0,0268+0,0081-\frac{490,34}{\mathrm{DOE}^{2}}$ & $0=0,0268+0,0066-\frac{490,34}{\mathrm{DOE}^{2}}$ & $0=0,0268+0,0058-\frac{490,34}{\mathrm{DOE}^{2}}$ \\
5 & $0=0,0242+0,0081-\frac{490,34}{\mathrm{DOE}^{2}}$ & $0=0,0242+0,0066-\frac{490,34}{\mathrm{DOE}^{2}}$ & $0=0,0242+0,0058-\frac{490,34}{\mathrm{DOE}^{2}}$ \\
$0=0,0258+0,0081-\frac{490,34}{\mathrm{DOE}^{2}}$ & $0=0,0258+0,0066-\frac{490,34}{\mathrm{DOE}^{2}}$ & $0=0,0258+0,0058-\frac{490,34}{\mathrm{DOE}^{2}}$
\end{tabular}

Cerne, Lavras, v. 19, n. 3, p. 451-459, jul./set. 2013 
custo total resultou da soma desses custos em função da densidade ótima de estradas.

Na Tabela 5, apresenta-se um resumo dos custos obtidos em relação às DOE específicas. Como pode ser visto, ao comparar com outros trabalhos, os valores obtidos de DOE (120m.ha-1) e DME (32,3m), apresentaram valores maiores para DOE e menores para DME. Souza (2001) obteve em seu estudo valores de DOE e DME para extração com skidder de 20,2m.ha-1 e 183 metros, respectivamente. MacDonagh (1994) encontrou valores de 87,14 m.ha-1 e 44,44 m.ha-1 para densidade de estradas e 87,50 e 112,50 metros de distância média de arraste. Os estudos citados como comparativos não possuíam características semelhantes ao trabalho realizado, pois essas pesquisas foram realizadas em plantio com áreas planas que permitia o uso da mecanização na colheita florestal, fato não possível nessas seis propriedades avaliadas.

Todas as áreas, com exceção da 5 (São José do Calçado), apresentaram maior densidade de estradas em relação ao ótimo desejado, em razão, principalmente, do

Tabela 5 - Análise dos custos obtidos pela densidade ótima em relação a atual para um cenário de produtividade de 180, 220, 250 $\mathrm{m}^{3}$ por hectare.

Table 5 - Cost analysis obtained by the optimal density in relation to the current scenario of a 180, 220, $250 \mathrm{~m}^{3}$ per hectare.

\begin{tabular}{|c|c|c|c|c|c|c|c|c|c|c|c|c|}
\hline Prop & $\begin{array}{c}\mathrm{DE} \\
\text { atual }\end{array}$ & DOE & DME & $\begin{array}{c}\text { Cestsec } \\
\text { atual }\end{array}$ & $\begin{array}{c}\text { Cestsec } \\
\text { ótimo }\end{array}$ & $\begin{array}{l}\text { Cpap } \\
\text { atual }\end{array}$ & $\begin{array}{l}\text { Cpap } \\
\text { ótimo }\end{array}$ & $\begin{array}{l}\text { Cext } \\
\text { atual }\end{array}$ & $\begin{array}{l}\text { Cext } \\
\text { ótimo }\end{array}$ & $\begin{array}{c}\mathrm{CT} \\
\text { atual }\end{array}$ & $\begin{array}{c}\mathrm{CT} \\
\text { ótimo }\end{array}$ & Benf \\
\hline \multicolumn{13}{|c|}{$180 \mathrm{~m}^{3} \cdot \mathrm{ha}^{-1}$} \\
\hline 1 & 373,9 & 127,4 & 30,41 & 3,03 & 1,03 & 8,26 & 2,79 & 1,76 & 4,33 & 13,05 & 8,15 & 4,90 \\
\hline 2 & 236,2 & 122,1 & 31,74 & 1,91 & 0,99 & 5,86 & 3,00 & 2,52 & 4,50 & 10,29 & 8,49 & 1,80 \\
\hline 3 & 269,9 & 118,5 & 32,69 & 2,19 & 0,96 & 7,23 & 3,15 & 2,26 & 4,62 & 11,68 & 8,73 & 2,95 \\
\hline 4 & 158,5 & 117,2 & 33,07 & 1,28 & 0,95 & 4,37 & 3,20 & 3,54 & 4,67 & 9,19 & 8,82 & 0,37 \\
\hline 5 & 111,6 & 123,2 & 31,45 & 0,90 & 1,00 & 2,70 & 2,95 & 4,84 & 4,46 & 8,44 & 8,41 & 0,04 \\
\hline 6 & 183,6 & 120,3 & 32,22 & 1,49 & 0,97 & 4,74 & 3,07 & 3,11 & 4,56 & 9,34 & 8,60 & 0,73 \\
\hline Média & 222,2 & 121,5 & 31,93 & 1,80 & 0,98 & 5,53 & 3,03 & 3,01 & 4,52 & 10,33 & 8,53 & 1,80 \\
\hline \multicolumn{13}{|c|}{$220 \mathrm{~m}^{3} \cdot \mathrm{ha}^{-1}$} \\
\hline 1 & 373,9 & 130,6 & 29,66 & 2,48 & 0,87 & 8,26 & 2,86 & 1,76 & 4,23 & 12,50 & 7,96 & 4,54 \\
\hline 2 & 236,2 & 124,9 & 31,02 & 1,57 & 0,83 & 5,86 & 3,07 & 2,52 & 4,40 & 9,95 & 8,30 & 1,65 \\
\hline 3 & 269,9 & 121,1 & 32,00 & 1,79 & 0,80 & 7,23 & 3,22 & 2,26 & 4,53 & 11,28 & 8,55 & 2,73 \\
\hline 4 & 158,5 & 120,0 & 32,38 & 1,05 & 0,79 & 4,37 & 3,28 & 3,54 & 4,57 & 8,96 & 8,64 & 0,32 \\
\hline 5 & 111,6 & 126,1 & 30,73 & 0,74 & 0,84 & 2,70 & 3,03 & 4,84 & 4,37 & 8,28 & 8,24 & 0,04 \\
\hline 6 & 183,6 & 123,0 & 31,51 & 1,22 & 0,82 & 4,74 & 3,15 & 3,11 & 4,46 & 9,07 & 8,43 & 0,64 \\
\hline Média & 222,2 & 124,2 & 31,22 & 1,47 & 0,82 & 5,53 & 3,10 & 3,01 & 4,43 & 10,01 & 8,35 & 1,65 \\
\hline \multicolumn{13}{|c|}{$250 \mathrm{~m}^{3} \cdot \mathrm{ha}^{-1}$} \\
\hline 1 & 373,9 & 132,5 & 29,25 & 2,18 & 0,77 & 8,26 & 2,90 & 1,76 & 4,18 & 12,20 & 7,85 & 4,35 \\
\hline 2 & 236,2 & 126,5 & 30,63 & 1,38 & 0,74 & 5,86 & 3,11 & 2,52 & 4,35 & 9,76 & 8,20 & 1,56 \\
\hline 3 & 269,9 & 122,6 & 31,61 & 1,57 & 0,72 & 7,23 & 3,26 & 2,26 & 4,47 & 11,06 & 8,45 & 2,62 \\
\hline 4 & 158,5 & 121,1 & 32,00 & 0,92 & 0,71 & 4,37 & 3,32 & 3,54 & 4,52 & 8,83 & 8,55 & 0,29 \\
\hline 5 & 111,6 & 127,8 & 30,33 & 0,65 & 0,75 & 2,70 & 3,07 & 4,84 & 4,31 & 8,19 & 8,13 & 0,07 \\
\hline 6 & 183,6 & 124,5 & 31,12 & 1,07 & 0,73 & 4,74 & 3,19 & 3,11 & 4,41 & 8,92 & 8,33 & 0,59 \\
\hline Média & 222,2 & 125,8 & 30,82 & 1,30 & 0,73 & 5,53 & 3,14 & 3,01 & 4,37 & 9,83 & 8,25 & 1,58 \\
\hline
\end{tabular}

Em que: Prop= Propriedade; $\mathrm{DE}=$ densidade atual de estradas na propriedade $(\mathrm{m} / \mathrm{ha}) ; \mathrm{DOE}=$ densidade ótima de estradas $\left(\mathrm{m} \cdot \mathrm{ha}^{-1}\right)$; $\mathrm{DME}=$ distância média de extração $(\mathrm{m})$; Cestsec.$=$ custo da estrada secundária $\left(\mathrm{R} \$ . \mathrm{m}^{-3}\right)$; Cpap=custo de perda de área produtiva $\left(\mathrm{R} \$ . \mathrm{m}^{-3}\right) ; \mathrm{Cext}=$ custo de extração $\left(\mathrm{R} \$ . \mathrm{m}^{-3}\right) ; \mathrm{CT}=$ custo total $\left(\mathrm{R} \$ . \mathrm{m}^{-3}\right) ; \mathrm{Benf}=$ Benefício.

Cerne, Lavras, v. 19, n. 3, p. 451-459, jul./set. 2013 
fato de que, anteriormente, as propriedades possuíam cultivos de lavouras de café que necessitavam de uma maior quantidade de estradas, para facilitar a aplicação de insumos e controle de plantas daninhas e colheita dos frutos. Já, a propriedade 5 apresentou uma densidade menor que o desejado, em decorrência das estradas serem construídas especificamente para o povoamento, visto que antes o terreno era destinado a pecuária, não sendo necessário uma maior quantidade de estradas.

Conforme é mostrado na Tabela 5 e para todas as propriedades analisadas, quando atingem o ótimo, os custos diminuem e, consequentemente, aumenta a satisfação (no aspecto financeiro) do produtor rural pelo empreendimento, conforme visualizado na Figura 2. Como pode ser visto, a DOE e a DE estão extremamente altas em relação à média brasileira de 90,10 m.ha ${ }^{-1}$ proposto por Machado (1989). Tal resultado deve-se ao fato de estarem, as propriedades estudadas, localizadas em relevo acidentado, assim necessitando de maior quantidade de estradas para facilitar a extração da madeira, que era realizada pelo método manual, visto que quanto menor a rede de estrada mais tempo se gasta para realizar o baldeio da madeira e, por consequência, maior será o custo para essa etapa. Assim é necessário um maior planejamento de construção de estradas por parte das empresas florestais fomentadoras e dos produtores, visando a minimizar a perda de área de efetivo plantio bem como agregar maior receita aos proprietários.

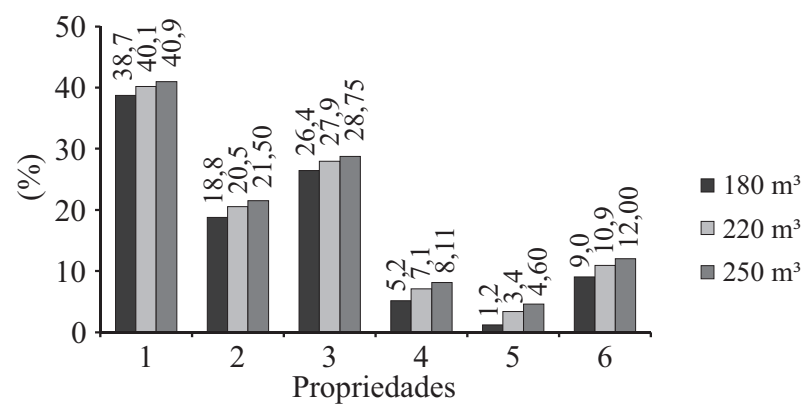

Figura 2 - Porcentagem de ganho do produtor por $\mathrm{m}^{3}$ de madeira quando atinge a densidade ótima.

Figure 2-Gain (\%) of the producer in cubic meters of wood when optimal road density is reached.

Na Figura 2, é mostrado que quanto maior a produtividade do povoamento florestal quando se atinge uma densidade ótima de estradas, maior são os benefícios para o produtor, tornando o empreendimento mais viável economicamente, em razão da redução dos valores de custos totais.

Cerne, Lavras, v. 19, n. 3, p. 451-459, jul./set. 2013
A propriedade 1 teve a maior variação de benefícios, pela relação da densidade atual e a ótima (média de 39,93\% para as três produtividades) ser alta, ou seja, apresenta mais estrada do que o necessário. Assim, ao atingir o ponto ótimo de estradas diminuem-se bastante os custos de perda de área produtiva e de estradas, reduzindo consideravelmente os custos totais. Já, a propriedade 5 apresentou a menor variação de ganho nos diferentes cenários de produtividade, pela sua densidade atual de estrada ser a mais próxima do ideal em relação às demais áreas avaliadas.

\section{CONCLUSÕES}

As áreas florestais pesquisadas, em sua grande maioria, possuem densidades de estradas muito acima do ótimo recomendado, mostrando a falta de conhecimentos e critérios no planejamento e aproveitamento dos plantios.

A elevada densidade de estradas nas propriedades rurais mostrou a necessidade de maior planejamento no dimensionamento dos talhões, possibilitando o maior aproveitamento da área de plantio e redução dos custos de produção.

Ao atingir a densidade ótima de estradas, os custos de estradas e de perda de áreas produtivas são reduzidos, aumentando a viabilidade econômica do empreendimento.

Além da densidade ótima de estradas é necessário que os produtores adotem tecnologias que possibilitem uma maior produtividade em suas áreas.

\section{REFERÊNCIAS}

ARCE, J. E. Um sistema de programação do transporte principal de multiprodutos florestais visando à minimização de custos. 1997. 134 f. Dissertação (Mestrado em Ciência Florestal) - Universidade Federal do Paraná, Curitiba, 1997.

BECKER, G. Optimization of road network and transport systems: a pre-condition for an improved organization and design of labour in forest. In: SEMINÁRIO DE ATUALIZAÇÃO SOBRE SISTEMAS DE COLHEITA DE MADEIRA E TRANSPORTE FLORESTAL, 8., 1994, Curitiba. Anais... Curitiba: UFPR/FUPEF, 1994. p. 111-115.

DIETZ, P. Parâmetros da rede viária e sua otimização. In: CURSO DE ATUALIZAÇÃO SOBRE SISTEMAS DE COLHEITA DE MADEIRA E TRANSPORTE FLORESTAL, 4., 1983, Curitiba. Anais... Curitiba: FUPEF, 1983. p. 22-35. 
EMPRESA BRASILEIRA DE PESQUISA AGROPECUÁRIA. Otimização da rede de estradas secundárias em projetos de manejo sustentável de floresta tropical. Brasília, 1997. (Circular Técnica).

HOSOKAWA, R. T.; MENDES, J. B. Planejamento florestal: técnicas para a manutenção da contribuição do setor florestal à economia nacional. Revista Floresta, Curitiba, v. 15, n. 1/2, p. 4-7, 1984.

LEFTWICH, R. H. O sistema de preços e a alocação de recursos. São Paulo: Pioneira, 1983. 154 p.

LOPES, E. S.; MACHADO, C. C.; SOUZA, A. P. Classificação e custos de estradas em florestas plantadas na região sudeste do Brasil. Revista Árvore, Viçosa, v. 26, n. 3 , p. 329-338, maio/jun. 2002.

MACHADO, C. C. Sistema brasileiro de classificação de estradas florestais (SIBRACEF): desenvolvimento e relação com o meio de transporte florestal rodoviário. 1989. 188 f. Tese (Doutorado em Ciências Florestais) - Universidade Federal do Paraná, Curitiba, 1989.

MACHADO, C. C.; LOPES, E. S. Análise da influência do comprimento de toras de eucalipto na produtividade e custo da colheita e transporte florestal. Cerne, Lavras, v. 6, n. 2, p. 124-129, 2000.

MALINOVSKI, J. R.; PERDONCINI, W. C. Estradas florestais. Irati: GTZ, 1990. 100 p.

MACDONAGH, P. M. A avaliação técnico-econômica da extração de Pinus spp. utilizando tratores com garra no sul do Brasil. 1994. 156 f. Dissertação (Mestrado em
Engenharia Florestal) - Universidade Federal do Paraná, Curitiba, 1994.

MOOSMAYER, H. Economia florestal. Curitiba: UFPR, 1967. $70 \mathrm{p}$.

PEREIRA, D. P. Análise técnica e de custos de povoamento de eucalipto sob reparo manual e mecanizado do solo em área declivosa no sul do Espírito Santo. 2010. 112 f. Dissertação (Mestrado em Ciências Florestais) - Universidade Federal do Espírito Santo, Jerônimo Monteiro, 2010.

PEREIRA NETO, S. D. Análise econômica da densidade de estradas nas áreas de produção de Eucalyptus. 1995. 133 f. Dissertação (Mestrado em Engenharia Florestal) Universidade Federal do Paraná, Curitiba, 1995.

SOUZA, D. O. Avaliação dos diferentes níveis de mecanização na atividade de colheita de madeira. Curitiba: UFPR/PIBIC/CNPq, 2001. 74 p. Relatório Técnico-Científico Final.

SPEIDEL, G. Economia florestal. Curitiba: UFPR, 1966. 167 p.

ZAGONEL, R. Análise da densidade ótima de estradas de uso florestal em relevo plano de áreas com produção de Pinus taeda. 2005. 100 f. Dissertação (Mestrado em Engenharia Florestal) - Universidade Federal do Paraná, Curitiba, 2005.

ZAGONEL, R.; CORREA, C. M. C.; MALINOVSKI, J. R. Densidade ótima de estradas de uso florestal em áreas de relevo plano em povoamentos de Pinus taeda no planalto catarinense. Scientia Forestalis, Piracicaba, v. 36, n. 77, p. 33-41, 2008.

Recebido: 23 de maio de 2011; aceito: 28 de fevereiro de 2013.

Cerne, Lavras, v. 19, n. 3, p. 451-459, jul./set. 2013 
